\title{
Simulacro de las pruebas internacionales: una mirada desde los estándares de lengua
}

\author{
Simulation of international tests: a look from the language standards \\ Simulação dos testes internacionais: um olhar a partir dos padrões da língua
}

Jaddy Brigitte Nielsen Nino*

\begin{abstract}
* PhD. en Educación con énfasis en investigación, coordinadora de internacionalización. Directora del grupo de investigación Inglés Como Mediación Cultural.
\end{abstract}

Cómo citar: Nielsen, J.B (2017) Simulacro de las pruebas internacionales: una mirada desde los estándares de la lengua. Sophia, 13(1): 55-63.

\section{Resumen}

El siguiente artículo tiene como objetivo principal comparar los resultados finales de las asignaturas de los cursos modulares y su relación con las pruebas internacionales correspondiente a cada nivel de acuerdo con el Marco Común Europeo que se ha establecido en la ruta académica del programa de licenciatura en inglés. Se parte del resultado inicial del curso intersemestral para la preparación de pruebas internacionales y así se puede determinar el dominio de una lengua extranjera del estudiante de la Licenciatura en Inglés de la Facultad de Ciencias de la Educación de la Universidad la Gran Colombia, sede Bogotá. Los resultados arrojados a través de pruebas post y pre demuestran que existen algunos factores que influyen en el bajo dominio de la lengua tales como falencias en conceptos básicos, la autonomía, motivación y tiempo de dedicación además el incremento en la deserción de los estudiantes, siendo un curso libre impartido por profesionales expertos. Se concluye, que los cursos intersemestrales para la preparación de una prueba internacional no basta con realizar simulaciones de dichos exámenes sino que generar unas metas claras que permitan concluir adecuadamente la ruta académica propuesta dentro del currículo.

Palabras clave: Aprendizaje, curso universitario, enseñanza, evaluación, pruebas estandarizadas.

The main goal of this article is to compare the final results of the subjects in the modular courses and their relation with the international tests corresponding to each level within the Common European Framework, according to the established in the academic route of the English teaching academic program (Licenciatura en Inglés). It starts from the initial results of the inter-semester preparation course for international tests, in order to determine the foreign language command of students in the English teaching academic program of the Faculty of Education Sciences, in La Gran Colombia University, Bogota. The results obtained through pre and post tests show that there are some factors responsible for low language skills, such as shortcomings in basic concepts, autonomy, motivation and dedication time, as well as the increase in students' dropout rates. It is concluded that for inter-semester courses, performing simulations is not enough; for that reason, it is necessary to establish definite goals intended to adequately conclude the proposed academic route in the curriculum.

Key words: Learning, teaching, evaluation, standardized tests, university subject. 


\section{Resumo}

O seguinte artigo tem por objetivo principal comparar os resultados finais das matérias dos cursos modulares e a sua relação com os testes internacionais correspondentes para cada nível de acordo com o Quadro Europeu Comum estabelecido no percurso acadêmico do programa da licenciatura em inglês. O ponto de partida é o resultado inicial do curso inter-semestral para a preparação de testes internacionais. Desta forma pode ser determinado o domínio de uma língua estrangeira por parte do estudante da licenciatura em inglês da Faculdade de Ciências da Educação da Universidade La Gran Colombia, com sede em Bogotá. Os resultados obtidos através de provas post e pré demonstram a existência de alguns fatores que influem em um fraco domínio da língua, tais como deficiências em conceitos básicos, autonomia, motivação e tempo de dedicação, além do crescimento no abandono escolar dos estudantes, sendo um curso livre fornecido por profissionais especializados. Se conclui, que nos cursos inter-semestrais para a preparação de um teste internacional, não é suficiente a realização de simulações das provas, é preciso gerar metas claras que permitam concluir devidamente o percurso acadêmico proposto no interior do currículo.

Palavras- chave: aprendizagem, ensino, avaliação, testes padronizados, curso universitário.

\section{Introducción}

Dentro de este artículo se hace necesario resaltar el rol de la competencia comunicativa porque ha sido relevante a través del tiempo, pero hoy en día ha generado mayor impacto, en tanto se constituye en la posibilidad de transmitir, interpretar mensajes y lograr la negociación del significado interpersonal con los contextos específicos, lo cual involucra aspectos como: el discurso, la interacción, lo pragmático y la negociación en la comunicación según Hymes (1962 citado por Nielsen 2014).

Con lo anterior, el aprendizaje- enseñanza y evaluación de una lengua extranjera entonces se ve no solo como un proceso potencial predecible del desarrollo de la competencia comunicativa sino también fundamental en la creación del significado en el que se involucra la negociación interactiva entre los estudiantes. De este modo, Canale (1983) define cuatro componentes o subcategorías de la competencia comunicativa. Organizada de la siguiente manera, la competencia lingüística, pragmática, estas dos categorizadas como parte del lenguaje sistemático, en cambio la competencia estratégica y sociolingüística hace parte de los aspectos funcionales de la lengua (el léxico, la fonética, la sintaxis, la semántica y la ortografía).

Por otro lado, los términos legales establecidos en el Marco Común Europeo de Referencia para las lenguas: aprendizaje, enseñanza, evaluación (Cadiz, 2002) incluye el proyecto general de política lingüística del Consejo de Europa, denominado de ahora en adelante MCER. El cual ha unificado las directrices específicas para el aprendizaje, la enseñanza y evaluación de lenguas dentro del contexto mundial:
Este esfuerzo queda suficientemente avalado por proyectos que han marcado de forma significativa en las últimas décadas, las líneas de trabajo de los profesionales de la enseñanza de lenguas, con el desarrollo de las escalas descriptivas de niveles lingüísticos para cada una de las habilidades. (Cadiz, citado por Nielsen 2014:294).

El MCER según Cadiz (2002) es un estamento legal que orienta los procesos de aprendizaje-enseñanza y evaluación de las lenguas extranjeras en el contexto mundial y nacional, en tanto provee de lineamientos curriculares que adoptan tanto las universidades públicas como privadas en el marco de los planes de mejoramiento propuestos por el Ministerio de Educación Nacional (MEN) dentro del Programa Nacional de Bilingüismo (2006) denominado PNB. Dicho plan retoma literalmente el MCER (2002) y lo adapta para establecer un objetivo común por medio de estándares curriculares en el contexto educativo colombiano: "Lograr ciudadanos y ciudadanas capaces de comunicarse en inglés, de tal forma que puedan insertar al país en los procesos de comunicación universal, en la economía global y en la apertura cultural, con estándares internacionalmente comparables" (PNB, 2006 citado en Nielsen 2015:298).

De este modo, el Plan Nacional de Bilingüismo en la actualidad, específicamente llamado Colombia Very Well (2015-2025) define unas metas específicas a nivel educativo universitario que van más allá de la autonomía y que es lograr estandarizar los niveles de dominio de lengua por medio del PNB 
(2006) en especial, los programas de licenciatura en lenguas extranjeras $\mathrm{y} / \mathrm{o}$ afines deben garantizar a la sociedad que han graduado licenciados competentes comunicativamente a partir de lo propuesto por el MCER Usuario C1. Esto muchas veces se logra demostrar a partir de exámenes estandarizados que permite dar una clasificación al usuario de lengua extranjera. Dichas pruebas no tienen en cuenta cuales fueron los riesgos, desafíos y retos que ha tenido el usuario dentro del proceso de aprendizaje-enseñanza de la lengua diferente a la materna, su enfoque principal es demostrar dentro de una sociedad el dominio de lengua logrado y que realmente certifique cuan competente es en dicha lengua extranjera.

\section{Revisión de la literatura}

Marco Común Europeo de Referencia para las Lenguas Extranjeras aprendizaje, enseñanza y evaluación denominado MCER (Cadiz, 2002), ha desarrollado un considerable esfuerzo por la unificación de directrices para el aprendizaje y la enseñanza de lenguas dentro del contexto europeo e incluso latinoamericano. Este esfuerzo queda suficientemente avalado por proyectos que han marcado de forma significativa en las últimas décadas las líneas de trabajo de los profesionales de la enseñanza de lenguas, como son el desarrollo de las escalas descriptivas de niveles lingüísticos: "[descritas en] el Nivel Umbral, Nivel Plataforma y Nivel avanzado, o en informes y trabajos de orientación como Transparencia y coherencia en el aprendizaje de lenguas en Europa, Objetivos para el aprendizaje de lenguas, entre otros" (Cadiz, 2002).

El MCER (2002) orienta los procesos de aprendizaje-enseñanza de las lenguas extranjeras en el contexto mundial y en el contexto colombiano ha sido adoptado. A partir del uso de la lengua, en función de activar la competencia comunicativa con el fin de consolidar los procesos que conllevan la expresión y establece a su vez las categorías necesarias para la descripción de las lenguas extranjeras según los parámetros descritos en el documento de acuerdo a los ámbitos y las situaciones que determinan el contexto del uso de la lengua, todo ello relacionado especialmente con las estrategias de aprendizaje (Oxford, 1990) y los canales de comunicación.

En la actualidad, el mundo exige que se domine una lengua extranjera, como bien lo retoma (Galindo, 2011) la afirmación del Rodolfo Suárez, director del departamento de Lenguas Extranjeras de la
Universidad Nacional de Colombia quien considera importante y esencial la habilidad de producción oral: "[conocer y perfeccionar otro idioma que no sea el nuestro] sin duda se trata de un valor agregado que permite el acceso a mejores oportunidades de trabajo o de estudio"(p.7). Debido a que el mundo exige que se domine más de una lengua, hay que tener en cuenta que dentro de los requisitos de varias de las instituciones internacionales y nacionales se exige conocimientos al menos una lengua extranjera a partir de los niveles intermedio y avanzado. De este modo, se considera fundamental el manejo de una lengua extranjera en nuestros tiempos pues esto a su vez permite el acceso a la información y circulación del conocimiento como elemento necesario para llegar a pensar en la producción del conocimiento global.

\section{Competencia comunicativa}

En el caso de las lenguas extranjeras se determinan las competencias por el intento de mantener la comunicación lo más auténtica y coherente posible. El término competencia comunicativa inicialmente ha sido propuesto por Hymes (1962). El autor sostiene que la competencia comunicativa nos permite transmitir, interpretar mensajes y lograr la negociación del significado interpersonal con los contextos específicos.

Además, desde las perspectivas del constructivismo social se resalta la lengua como un medio de comunicación entre individuos. Más adelante, Hymes (1972) involucra aspectos como: el discurso, la interacción, lo pragmático y la negociación entre otras en la comunicación. Incluso, profesores y editores de textos consideran que la lengua es utilizada dentro del salón de clases como un lugar significativo con intercambios auténticos entre usuarios de la lengua objeto.

A partir de ello, el aprendizaje de una lengua extranjera comienza a verse no solo como un proceso potencial predecible del desarrollo sino como la creación del significado a través de la negociación interactiva entre aprendices. Canale (1983) inicialmente definió cuatro componentes o subcategorías para realizar la construcción de la competencia comunicativa.

Las dos primeras subcategorías reflejan el uso del lenguaje sistemático entre sí competencia lingüística y pragmática; y las dos últimas definen los aspectos funcionales de la comunicación competencia sociolingüística y estratégica. La competencia lingüística, hace referencia a los elementos formales 
de la lengua, la cual incluye los conocimientos y destrezas sobre el léxico, la fonética, la sintaxis, la semántica y la ortografía. Además, por su carácter de base, permite su aplicación a una variedad de situaciones diferentes. La competencia pragmática, permite hacer un uso práctico de los recursos lingüísticos. Esta se divide en competencia discursiva, que es la que permite hacer secuencias de oraciones para producir extensas expresiones textuales; y la competencia funcional, que se emplea para conocer la función de las formas lingüísticas y de cómo estas se relacionan en situaciones comunicativas reales. La competencia sociolingüística según Hudson (1980), tiene que ver con el conocimiento de las condiciones sociales y culturales que son parte de la lengua que se va a aprender. Entre ellas están las normas de cortesía, las relaciones respecto de la edad, el género y el estatus social, entre otros.

Para Savigon (1983, citado por Brown, 2000) define la competencia comunicativa como algo relativo pero no absoluto y depende de la colaboración de todos los participantes que hacen parte de ella, en este caso a partir de todos y cada uno de los sujetos quienes se están comunicando. Esto le permite enriquecerse y por ser abordada en distintos contextos se enmarca dentro de aspectos éticos, estéticos, sociales y culturales. Ahora bien, para enmarcar la importancia de la competencia comunicativa según Hymes (1972) el principal objetivo es intentar desarrollar la capacidad de usar los conocimientos adquiridos de una lengua meta, en este caso del inglés como lengua extranjera, al propiciarse el uso dentro y fuera del aula.

\section{Relación entre aprender y enseñar lenguas extranjeras}

Según la indagación teórica de Vez (2001), en las décadas de los ochenta y noventa simplemente se evaluaba el aprendizaje, a partir de los logros obtenidos de acuerdo con la asimilación del conocimiento, pero en la actualidad se puede asegurar que un aula de clase no sólo se limita a este aspecto sino por el contrario los estudiantes aprenden por medio de instrumentos de socialización, poder y comunicación indispensables en la sociedad de la información en la que nos encontramos "hoy las lenguas se aprenden, y se mejoran sus usos, porque solo así podemos convertirnos en comunicadores sociales, capaces de competir en una sociedad de cambio permanente donde los retos son más demandantes y funcionales" (Vez, 2001:316), esta afirmación puede asegurar que muy pocas veces se había evidenciado tal situación cuando las lenguas simplemente se enseñaban.

Es así que el consenso progresivo de correlacionar el acto de enseñar y el acto de aprender una lengua extranjera permite fácilmente a los docentes y estudiantes ahondar en la competencia comunicativa de forma efectiva (Nielsen, 2012). Por medio de la lengua como recurso e instrumento de comunicación, de construcción y de negociación de significados tanto a nivel personal como social, se puede hablar de proceso coherente y eficaz de aprendizaje-enseñanza.

Retomando organizaciones internacionales en educación, el aprendizaje y la enseñanza de la lengua extranjera son actos sociales, que dependen de las relaciones con los demás, el contexto, el entorno, el mundo y uno mismo. Dicho aprendizaje y enseñanza son interesantes, generando curiosidad por la vida y la confianza necesaria para iniciar nuevas interacciones sociales en una lengua extranjera.

\section{Materiales y métodos}

Inicialmente, surge una inquietud por parte de un grupo de profesores del programa de Licenciatura en Inglés de las jornadas mañana y tarde de la Facultad de Ciencias de la Educación de la Universidad La Gran Colombia, sede Bogotá, frente a los resultados arrojados en los cursos modulares, es decir los cursos de lengua básicos que permiten afianzar el dominio de la lengua extranjera en este caso, el inglés de estudiantes adscritos, debido a que en su gran mayoría no alcanzan los mínimos requeridos establecidos en el plan de estudios que tomando como referencia el Marco Común Europeo - MCER.

Por tal motivo, se determina que las notas entregadas durante ese semestre no se limitaran a un cómputo numérico para llegar a un simple resultado final como lo es "pasar o perder". Debido a que los contenidos de enseñanza utilizados para el aprendizaje de una lengua distinta a la materna en estudiantes universitarios deben realizarse de manera constante e ir más allá de un cronograma institucional programado. Con todo lo anterior, surge una pregunta ¿Cómo pueden los miembros académicos de la universidad apoyar el proceso académico de los estudiantes de Licenciatura en Inglés?

Para responder el interrogante mencionado se partió de una clasificación de los resultados finales de los estudiantes en las notas de los cursos modulares computando el tercer corte, para luego crear cursos 
inter-semestrales totalmente gratis dirigidos a los estudiantes que presentan dificultades y bajos resultados en el momento de presentar simulacros en las pruebas internacionales establecidas durante el semestre.

Es importante resaltar que los cursos de refuerzo en el intersemestral, estaban dirigidos a los estudiantes que pasaron el curso académico estipulado en el plan de estudios denominado: Breakthrough, PreIntermediate-Intermediate-Upper Y Advanced, pero perdieron el simulacro que se propone en cada curso.

Para ello, se dispuso de un horario del que cada curso daría inicio desde el jueves 5 de junio y terminaría el 26 del mismo mes, finalizando con nuevamente un simulacro de examen internacional, en el siguiente horario: Mañana 9:00 /12:00 y Noche: 6:00/9:00. Vale la pena resaltar que se trabajaron los días martes y jueves, con una intensidad horaria de 6 horas a la semana. Con esto cada estudiante recibió 24 horas de capacitación para responder en forma significativa con las exigencias del siguiente nivel estipulado en la ruta académica del programa al cual el estudiante se inscribiría al siguiente semestre, horas brindadas por la universidad acompañadas por 10 docentes en aras de fortalecer el proceso de afianzamiento de los estudiantes quienes serán futuros licenciados de inglés y que en algún momento de sus vidas presentaran pruebas internacionales que certifiquen su nivel de domino.

Tabla 1. Distribución de los docentes dependiendo del grupo y nivel establecido en el plan de estudios del programa de licenciatura.

\begin{tabular}{|c|c|c|}
\hline Curso Modular & $\begin{array}{l}\text { Docente } \\
\text { mañana }\end{array}$ & $\begin{array}{c}\text { Docente } \\
\text { noche }\end{array}$ \\
\hline Breakthrough & üüüü & üüüü \\
\hline Pre-Intermediate & üüüü & üüüü \\
\hline Intermediate & üüüü & üüüü \\
\hline $\begin{array}{l}\text { Upper- } \\
\text { Intermediate }\end{array}$ & Docente 4 & üüüü \\
\hline Advanced & üüüü & Docente 10 \\
\hline
\end{tabular}

A su vez, los cursos inter-semestrales no superaban un número de veinte (20) estudiantes, permitiéndole a los docentes generar estrategias específicas según las necesidades de los estudiantes puesto que normalmente estos cursos modulares están conformados entre 28 y 37 estudiantes aproximadamente.

\section{Resultados}

Como se nombró, los cursos intersemestrales para la presentación de simulacros de exámenes internacionales se realizaron del 5 al 26 de junio, con el objetivo de ofrecerles una ayuda adicional a los estudiantes del Programa de Licenciatura en Inglés que aprobaron los niveles de inglés correspondientes a sus semestres académicos (breakthrough, Preintermediate, intermediate, upper-intermediate y advanced), pero que no obtuvieron un resultado satisfactorio en el desempeño del simulacro de la prueba internacional de inglés, determinado como examen final en cada curso modular. Es necesario resaltar que se estableció que durante el primer semestre académico los estudiantes debían obtener un puntaje mínimo de $70 \%$ para considerar el simulacro aprobada. Sin embargo, se dieron muchos casos de estudiantes que aprobaron el semestre a pesar de no sacar un buen resultado (igual o superior al 70\%) en el simulacro del examen internacional.

Los cursos intersemestrales no eran cursos formales, ya que no eran equivalentes a ningún espacio académico del programa y tampoco otorgaban ninguna nota o crédito académico a los estudiantes. Por lo tanto, ellos fueron invitados por sus profesores a asistir de manera voluntaria con el único objetivo de mejorar sus resultados en los simulacros de los exámenes internacionales. Los estudiantes que asistieron la totalidad del curso tuvieron la oportunidad de finalizar el proceso correspondiente y los resultados fueron socializados y analizados con el objetivo de simplemente entregar un informe al comité de facultad, que luego dentro del proceso investigativo se contrastaron los datos que obtuvieron los estudiantes en los cursos de preparación intersemestral con los resultados que habían obtenido previamente durante el semestre académico.

Es necesario aclarar que estos dos simulacros tenían el mismo formato según la prueba internacional, pero contenían preguntas totalmente diferentes, ya que se buscaba que los estudiantes no repitieran las preguntas que habían tomado antes. 
Los estudiantes fueron divididos en diferentes cursos según el nivel de dominio de la lengua y el curso modular que habían tomado durante el semestre académico, así:

Tabla 2. Diseño curricular de las asignaturas según el MCER. Fuente: Programa de Licenciatura en inglésFacultad de Ciencias de la Educación

\begin{tabular}{lcc}
\hline $\begin{array}{c}\text { Grupo de } \\
\text { estudiantes }\end{array}$ & $\begin{array}{c}\text { Tipo de prueba } \\
\text { internacional en la que } \\
\text { se prepararon los } \\
\text { estudiantes }\end{array}$ & $\begin{array}{c}\text { Nivel según el } \\
\text { MCER }\end{array}$ \\
\hline $\begin{array}{l}\text { Breakthrough } \\
\text { Preintermediate }\end{array}$ & KET & A2 \\
$\begin{array}{l}\text { Intermediate } \\
\text { Upper- } \\
\text { intermediate } \\
\text { Advanced }\end{array}$ & PET & B1 \\
\hline
\end{tabular}

En las siguientes dos tablas se muestra el número a nivel general de los estudiantes que se inscribieron en el curso intersemestral, la relación frente a los cambios que surgieron durante el tiempo que se desarrolló de acuerdo con las exigencias estipuladas para la inscripción de los mismos.

Tabla 3. Relación de las reacciones de los estudiantes frente a los cursos intersemestrales

\begin{tabular}{cccc}
\hline Inscritos & Desertaron & Finalizaron & Sin fallas \\
127 & 78 & 49 & 27 \\
\hline
\end{tabular}

Gráfico 1. Relación de las reacciones de los estudiantes frente a los cursos inter-semestrales.

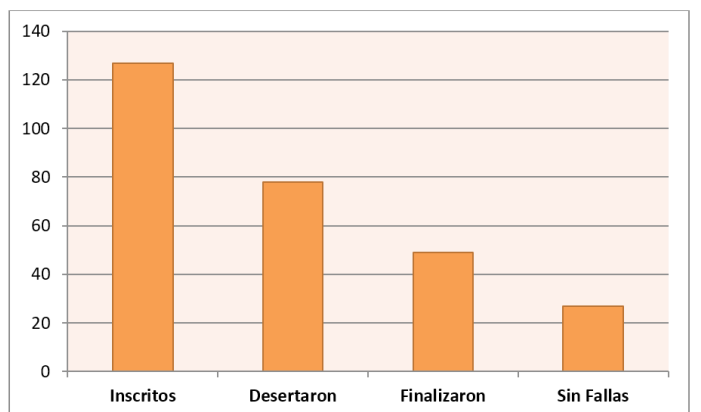

Fuente: Programa de Licenciatura en Inglés- Facultad de Ciencias de la Educación
En el gráfico 1 se presenta la evidencia de los resultados obtenidos de acuerdo con los estudiantes frente al primer simulacro del examen internacional que presentaron al finalizar el semestre académico (pre) y los resultados que obtuvieron en el segundo simulacro del examen internacional que realizaron en el curso inter-semestral (post). Tomando como referencia el nivel en el que estaban adscritos.

Gráfico 2. Comparación de los resultados pre-test y post-test de acuerdo con el nivel de dominio de lengua en que se encontraban los estudiantes.

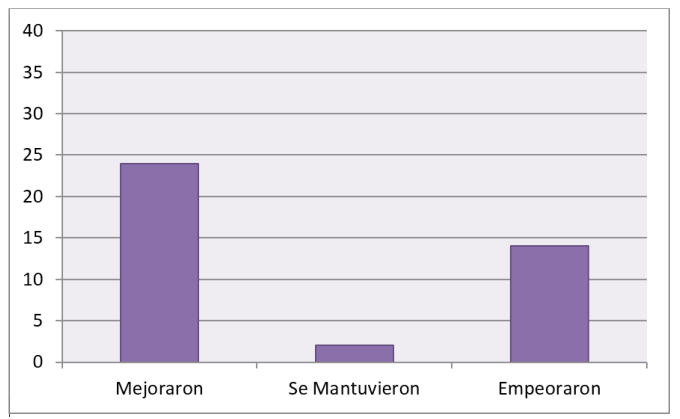

Fuente: Programa de Licenciatura en Inglés- Facultad de Ciencias de la Educación

Para una mayor precisión de los resultados arrojados anteriormente se describe en detalle por docente los resultados que se arrojaron en el proceso de la preparación para el simulacro de los exámenes internacionales durante los cursos inter-semestrales. Es importante aclarar que los datos numéricos son medidos por porcentajes, es decir, que el equivalente al $100 \%$ un resultado satisfactorio, $50 \%$ un resultado medio y $20 \%$ un resultado deficiente.

\section{Examen: KET- Breakthrough}

El resultado se presenta a partir de veintisiete (27) inscritos de los cuales veinte (20) desertaron del proceso y siete (7) estudiantes finalizaron pero solo tres (3) de ellos asistieron al curso completo. Discriminado de la siguiente manera: en la mañana tres estudiantes mejoraron y uno empeoró, en cambio un estudiante mejoró pero dos empeoraron, representado gráficamente así: 


\begin{tabular}{cccc}
\hline & Estudiante & $\begin{array}{c}\text { Puntaje } \\
\text { Pre-test }\end{array}$ & $\begin{array}{c}\text { Puntaje } \\
\text { Pos-test }\end{array}$ \\
\cline { 2 - 4 } KET Jornada & $\mathrm{A}$ & 43 & 51 \\
Mañana- & $\mathrm{B}$ & 60 & 56 \\
(Docente 1) & $\mathrm{C}$ & 43 & 48 \\
& $\mathrm{D}$ & 56 & 62 \\
\cline { 2 - 4 } & $\mathrm{E}$ & 56 & 54 \\
KET Jornada & $\mathrm{F}$ & 64 & 54 \\
Mañana & & 58 & 64 \\
(Docente 6) & $\mathrm{G}$ & 58 \\
\hline
\end{tabular}

\section{Examen: PET- Pre intermediate}

El resultado en este nivel se estableció a partir de la inscripción inicial de ocho (8) estudiantes de los cuales cuatro (4) de ellos desertaron del proceso, esto indica que la mitad de los participantes finalizaron el proceso pero solo dos (2) de ellos se reportan con una asistencia del $100 \%$. Conforme a lo anterior en la mañana un estudiante mejoró y uno empeoró, en cambio en la noche los dos estudiantes inscritos mejoraron sus resultados.

\begin{tabular}{cccc}
\hline & Estudiante & $\begin{array}{c}\text { Puntaje } \\
\text { Pre-test }\end{array}$ & $\begin{array}{c}\text { Puntaje } \\
\text { Pos-test }\end{array}$ \\
\cline { 2 - 4 } $\begin{array}{c}\text { PET Jornada } \\
\text { Mañana- } \\
\text { (Docente 2) }\end{array}$ & $\mathrm{H}$ & 62 & 70 \\
\cline { 2 - 4 } & $\mathrm{I}$ & 62 & 52 \\
\hline $\begin{array}{c}\text { PET Jornada } \\
\text { Mañana } \\
\text { (Docente 7) }\end{array}$ & $\mathrm{J}$ & 56 & 62 \\
\hline
\end{tabular}

\section{Examen: PET- Intermedio}

El resultado en este nivel indica que se inscribieron dieciocho (18) estudiantes pero once (11) de ellos desertaron del proceso, indicando que siete (7) finalizaron el curso pero solo dos (2) de ellos culminaron el proceso asistiendo a todas las sesiones programas. Esto también indica que en la jornada mañana dos (2) estudiantes mejoraron pero tres (3) empeoraron, en cambio en la noche un (1) estudiante mejoró y uno (1) empeoró.

\begin{tabular}{cccc}
\hline & Estudiante & $\begin{array}{c}\text { Puntaje } \\
\text { Pre-test }\end{array}$ & $\begin{array}{c}\text { Puntaje } \\
\text { Pos-test }\end{array}$ \\
\cline { 2 - 4 } PET Jornada & $\mathrm{L}$ & 58 & 40 \\
Mañana- & $\mathrm{M}$ & 32 & 40 \\
(Docente 3) & $\mathrm{N}$ & 56 & 47 \\
& $\tilde{\mathrm{N}}$ & 33 & 25 \\
& $\mathrm{O}$ & 55 & 61 \\
\cline { 2 - 4 } PET Jornada & $\mathrm{P}$ & 65 & 58 \\
Mañana- & $\mathrm{Q}$ & 65 & 70 \\
(Docente 8) & $\mathrm{Q}$ & &
\end{tabular}

\section{Examen: FCE- Upper-Intermediate}

El resultado arrojado en este nivel indica que hubo veintiséis (26) inscritos inicialmente pero se retiraron trece (13) estudiantes de los cuales la mitad de inscritos finalizó el curso pero solo 3 (tres) de ellos culminaron por completo su proceso asistiendo a todas las sesiones de clase programada. Si se analiza los datos por jornada se puede determinar que en la mañana cinco (5) estudiantes mejoraron y uno (1) empeoró, pero aparece una nueva variable en este nivel porque dos (2) estudiantes se mantuvieron con el mismo puntaje inicial. En cambio en la noche, cuatro (4) estudiantes mejoraron y uno (1) empeoró.

\begin{tabular}{cccc}
\hline & Estudiante & $\begin{array}{c}\text { Puntaje } \\
\text { Pre-test }\end{array}$ & $\begin{array}{c}\text { Puntaje } \\
\text { Pos-test }\end{array}$ \\
\cline { 2 - 4 } & $\mathrm{R}$ & 64 & 60 \\
FCE Jornada & $\mathrm{S}$ & 70 & 70 \\
Mañana- & $\mathrm{T}$ & 46 & 50 \\
(Docente 4) & $\mathrm{U}$ & 44 & 46 \\
& $\mathrm{~V}$ & 46 & 66 \\
& $\mathrm{~W}$ & 56 & 56 \\
& $\mathrm{X}$ & 54 & 60 \\
& $\mathrm{Y}$ & 58 & 66 \\
\cline { 2 - 4 } FCE Jornada & $\mathrm{Z}$ & 68 & 82 \\
Mañana- & $\mathrm{AA}$ & 64 & 78 \\
(Docente 9) & $\mathrm{AB}$ & 60 & 58 \\
& $\mathrm{AC}$ & 50 & 58 \\
\hline
\end{tabular}

\section{Examen: FCE-Advanced level}

\section{Jornada mañana}

El resultado se estableció a partir de diez (10) estudiantes inscritos de los cuales uno (1) solamente deserto del proceso, además en comparación con los otros niveles ocho (8) de los estudiantes que caminaron el proceso no obtuvieron ninguna inasistencia dentro del curso programado. Con lo anterior, se puede determinar que en la jornada de la mañana todos los estudiantes mejoraron y ninguno empeoró, en contraste con la jornada tarde en donde todos los estudiantes empeoraron de acuerdo al resultado arrojado. 


\begin{tabular}{cccc} 
& Estudiante & $\begin{array}{c}\text { Puntaje } \\
\text { Pre-test }\end{array}$ & $\begin{array}{c}\text { Puntaje } \\
\text { Pos-test }\end{array}$ \\
\cline { 2 - 4 } FCE Jornada & AE & 56 & 73 \\
Mañana- & AF & 46 & 74 \\
(Docente 5) & $\mathrm{AG}$ & 53 & 73 \\
& $\mathrm{AH}$ & 66 & 88 \\
& $\mathrm{AI}$ & 60 & 72 \\
\cline { 2 - 4 } & $\mathrm{AJ}$ & 40 & 54 \\
FCE Jornada & $\mathrm{AK}$ & 30 & 54 \\
Mañana- & $\mathrm{AL}$ & 40 & 40 \\
(Docente 10) & $\mathrm{AM}$ & 54 & 54 \\
\hline
\end{tabular}

Finalmente, se puede afirmar que hubo una mejoría por parte de los estudiantes que culminaron el proceso pero aun así no cumplen a cabalidad con el nivel establecido en el semestre académico en el que se encuentran. A su vez, influyen otros factores y es la importancia de crear en los estudiantes una autorregulación y disciplina para lograr el aprendizaje de una lengua extranjera, en este caso inglés. A pesar que el curso se oferto de forma voluntaria y sin generar costo alguno se evidencia que más del $70 \%$ de los estudiantes desertaron del proceso dando prioridad a otras actividades como por ejemplo: las vacaciones de fin de mitad de año o simplemente ocupar el tiempo en actividades laborales.

\section{Discusión de resultados}

Los resultados que obtuvieron los estudiantes no demostraron que existiera una mejora significativa. Solamente veinte-cuatro (24) de ellos mejoraron los resultados lo que equivale al $60 \%$. Por lo tanto, el porcentaje permanece bajo para aceptar una hipótesis que demuestre a la comunidad educativa mejores resultados debido al ofrecimiento de los cursos de preparación de exámenes internacionales en un periodo inter-semestral.

Igualmente, existen diferentes factores que pueden contribuir a que un gran porcentaje de estudiantes $(40 \%)$ no hayan mejorado o empeorado el resultado en la presentación de los exámenes internacionales. Por ejemplo, hay que tener en cuenta que los estudiantes fueron preparados para un simulacro de un examen internacional que ya conocían previamente. Sin embargo, las pruebas no miden el conocimiento sus conocimientos como usuarios de una lengua distinta a la materna, sino su nivel de competencia en inglés. Por lo tanto, se debe trabajar en mejorar el nivel de inglés de los estudiantes mediante talleres o cursos que se enfoquen en habilidades específicas (listening, writing, reading, speaking, grammar) y que les permita mejorar su nivel de dominio, para lograr ser profesionales competentes, sin limitarse simplemente preparar a los estudiantes para el simulacro de pruebas internacionales.

Finalmente, la deserción en los cursos fue alta, de hecho solo un $30 \%$ de los estudiantes tomó el simulacro del examen internacional programado y menos de un $20 \%$ asistió sin fallas. Esto podría ser un indicador de falta de motivación, porque era de manera voluntaria.

Este análisis fue realizado desde la mirada de los futuros licenciados en inglés, los cuales demuestran que los procesos académicos no se pueden limitar dentro de un horario estipulado, sino que además se debe enfocar en las necesidades de los estudiantes, en este caso mejorar su nivel de dominio en lengua durante los cursos modulares previos. El uso adecuado del inglés en estos futuros licenciados es un requerimiento en la actualidad a nivel laboral y social, pero a su vez demuestra la importancia como formadores de formadores la necesidad de generar estrategias de enseñanza propicias para alcanzar los objetivos propuestos según los estándares del MCER y por ende del MEN.

\section{Conclusiones}

En síntesis, ser competente en una lengua extranjera, en especial en inglés en Colombia es una tarea compleja debido a que implica propósitos distintos a nivel social, cultural y económico en el que no solo involucran el conocimiento de las reglas gramaticales dentro de un discurso oral o escrito, sino que también en contextos específicos de acuerdo con los roles de cada participante, las relaciones y el entorno en el cual se está inmerso.

Las recomendaciones pueden ir dirigidas a tomar este trabajo como base para posibles nuevos proyectos investigativos que permitan evidenciar la incidencia de otras variables en el uso de los simulacros para las pruebas internacionales y su incidencia en el aprendizaje-enseñanza-evaluación, y las maneras como desde la práctica pedagógica y las verdadera presentación de las pruebas internacionales junto con las estrategias que se generen por parte de los agentes implicados podrían ser promovidas para la educación. 


\section{Referencias bibliográficas}

Bachman, L. (1990). Fundamental Considerations in Language Testing. New York, USA: Oxford University Press.

Brown. (2000). Principles of language learning and teaching. San Francisco, USA: Pearson Education.

Cadiz, J. (2002). Marco Común Europeo de Referencia para las Lenguas: Aprendizaje, enseñan$z a$, evaluación. Madrid, Epaña: Subdirección General de Cooperación Internacional.

Canale, M. (1983). Theoretical bases of communicative approaches to second language teaching and testing. Applied Linguistics, 1-47.

Canale \& Swain. (1980). Theoretical bases of communicative approaches to Second Language Teaching and Testing. Linguistics, Applied, $1-47$.

Galindo, A. (2011). Los idiomas marcan la diferencia. Revista de Bilingüismo, 32-33.

Hymes, D. (1962). The ethnography of speaking. In T. \&. Gladwin, Antrhropology and Human Behavior (pp. 13-53). Washington D.C.: Antropological Society of Washington.

(1972). On Communicative Competence. En J. Pride, Sociolinguistics (págs. 269-293). Harmondsworth: Penguin Books.
Hudson, R. (1980). Sociolinguistics. Cambridge: Cambridge University Press.

MEN (2006). Estándares Básicos de Competencias en Lenguas Extranjeras: inglés (Vol. Serie número 22). Bogotá, Colombia: Revolución Educativa Colombia aprende.

Nielsen. J (2012). Estrategias de aprendizaje de las lenguas extranjeras en la educación superior. Simposio internacional de pedagogía, educación y humanidades, escuela y pedagogía transformadora (pág. 7). Cali, Colombia: Redipe.

(2014). Una Revisión de las estrategias que se usan para la enseñanza de las lenguas en programas de licenciatura. Entramados, 293-311.

Oxford, R. (1990). Language Learning Strategies. Estados Unidos: Heinle \& Heinle publishers.

PNB. (2006). Programa Nacional de Bilingüismo. Inglés como lengua extranjera: Una estrategia para la Competividad. Bogotá: MEN.

Vez, J. (2001). Formación en Didáctica de la Lenguas Extranjeras. Santa Fe, Argentina: Homo Sapiens Ediciones.

Weinstein. (1988). Assessing learning strategies. Learning and Studying Strategies, Academic Press N.Y. 